\title{
Editor's notes: As open as possible and as closed as needed
}

Welcome to the third issue of volume 43 of the IASSIST Quarterly (IQ 43:3, 2019).

Yes, we are open! Open data is good. Just a click away. Downloadable $24 / 7$ for everybody. An open government would make the decisionmakers' data open to the public and the opposition. As an example, communal data on bicycle paths could be open, so more navigation apps would flourish and embed the information in maps, which could suggest more safe bicycle routes. However, as demonstrated by all three articles in this IQ issue, very often research data include information that requires restrictions concerning data access. The second paper states that data should be 'as open as possible and as closed as needed'. This phrase originates from a European Union Horizon 2020 project called the Open Research Data Pilot, in 'Guidelines on FAIR Data Management in Horizon 2020 ' (July 2016). Some data need to be closed and not freely available. So once more it shows that a simple solution of total openness and one-size-fits-all is not possible. We have to deal with more complicated schemes depending on the content of data. Luckily, experienced people at data institutions are capable of producing adapted solutions.

The first article 'Restricting data's use: A spectrum of concerns in need of flexible approaches' describes how data producers have legitimate needs for restricting data access for users. This understanding is quite important as some users might have an automatic objection towards all restrictions on use of data. The authors Dharma Akmon and Susan Jekielek are at ICPSR at the University of Michigan. ICPSR has been a U.S. research archive since 1962, so they have much practice in long-term storage of digital information. From a short-term perspective you might think that their primary task is to get the data in use and thus would be opposed to any kind of access restrictions. However, both producers and custodians of data are very well aware of their responsibility for determining restrictions and access. The caveat concerns the potential harm through disclosure, often exemplified by personal data of identifiable individuals. The article explains how dissemination options differ in where data are accessed and what is required for access. If you are new to IASSIST, the article also gives an excellent short introduction to ICPSR and how this institution guards itself and its users against the hazards of data sharing.

In the second article 'Managing data in cross-institutional projects', the reader gains insight into how FAIR data usage benefits a cross-institutional project. The starting point for the authors - Zaza Nadja Lee Hansen, Filip Kruse, and Jesper Boserup Thestrup - is the FAIR principles that data should be: findable, accessible, interoperable, and re-useable. The authors state that this implies that the data should be as open as possible. However, as expressed in the ICPSR article above, data should at the same time be as closed as needed. Within the EU, the mention of GDPR (General Data Protection Regulation) will always catch the attention of the economical responsible at any institution because data breaches can now be very severely fined. The authors share their experience with implementation of the FAIR principles with data from several cross-institutional projects. The key is to ensure that from the beginning there is agreement on following the specific guidelines, standards and formats throughout the project. The issues to agree on are, among other things, storage and sharing of data and metadata, responsibilities for updating data, and deciding which data format to use. The benefits of FAIR data usage are summarized, and the article also describes the crossinstitutional projects. The authors work as a senior consultant/project manager at the Danish National Archives, senior advisor at The Royal Danish Library, and communications officer at The Royal Danish Library. The cross-institutional projects mentioned here stretch from Kierkegaard's writings to wind energy.

1/2 Rasmussen, Karsten Boye (2019) Editor's notes: As open as possible and as closed as needed, IASSIST Quarterly 43(3), pp. 1-2. DOI https://doi.org/10.29173/iq965 
While this issue started by mentioning that ICPSR was founded in 1962, we end with a more recent addition to the archive world, established at Qatar University's Social and Economic Survey Research Institute (SESRI) in 2017. The paper 'Data archiving for dissemination within a Gulf nation' addresses the experience of this new institution in an environment of cultural and political sensitivity. With a positive view you can regard the benefits as expanding. The start is that archive staff get experience concerning policies for data selection, restrictions, security and metadata. This generates benefits and expands to the broader group of research staff where awareness and improvements relate to issues like design, collection and documentation of studies. Furthermore, data sharing can be seen as expanding in the Middle East and North Africa region and generating a general improvement in the relevance and credibility of statistics generated in the region. Again, the FAIR principles of findable, accessible, interoperable, and re-useable are gaining momentum and being adopted by government offices and data collection agencies. In the article, the story of SESRI at Qatar University is described ahead of sections concerning data sharing culture and challenges as well as issues of staff recruitment, architecture and workflow. Many of the observations and considerations in the article will be of value to staff at both older and infant archives. The authors of the paper are the senior researcher and lead archivist at the archive of the Qatar University Brian W. Mandikiana, and Lois Timms-Ferrara and Marc Maynard - CEO and director of technology at Data Independence (Connecticut, USA).

Submissions of papers for the IASSIST Quarterly are always very welcome. We welcome input from IASSIST conferences or other conferences and workshops, from local presentations or papers especially written for the IQ. When you are preparing such a presentation, give a thought to turning your one-time presentation into a lasting contribution. Doing that after the event also gives you the opportunity of improving your work after feedback. We encourage you to login or create an author login to https://www.iassistquarterly.com (our Open Journal System application). We permit authors 'deep links' into the IQ as well as deposition of the paper in your local repository. Chairing a conference session with the purpose of aggregating and integrating papers for a special issue IQ is also much appreciated as the information reaches many more people than the limited number of session participants and will be readily available on the IASSIST Quarterly website at https://www.iassistquarterly.com. Authors are very welcome to take a look at the instructions and layout:

\section{https://www.iassistquarterly.com/index.php/iassist/about/submissions}

Authors can also contact me directly via e-mail: kbr@sam.sdu.dk. Should you be interested in compiling a special issue for the IQ as guest editor(s) I will also be delighted to hear from you.

Karsten Boye Rasmussen - September 2019 